\title{
A fullerene-carbene adduct as a crystalline molecular rotor: remarkable behavior of a spherically-shaped rotator $\dagger$
}

\author{
Andreas Lorbach, $\star^{* a}$ Emily Maverick, ${ }^{\mathrm{b}}$ Abel Carreras, ${ }^{\mathrm{bc}}$ Pere Alemany, ${ }^{\mathrm{c}}$ Guang Wu, ${ }^{\mathrm{a}}$ \\ Miguel A. Garcia-Garibay*b and Guillermo C. Bazan*a
}

\begin{abstract}
A new fullerene structure was recently obtained from the reaction of a Lewis basic $\mathrm{N}$-heterocyclic carbene $(\mathrm{NHC})$ and the Lewis acidic $\mathrm{C}_{60}$. The molecular features of the zwitterionic adduct can be described as a molecular rotor with the fullerene cage acting as the rotator that spins about one distinct axis given by its $\mathrm{C}-\mathrm{C}$ single bond linkage with the imidazolium heterocycle stator. A detailed structural analysis of the compound by means of single-crystal X-ray diffraction (XRD) revealed significant differences in the packing motifs of solvent-free and solvent-containing crystals. Variable temperature single-crystal XRD experiments ( $80 \mathrm{~K} \leq T \leq 480 \mathrm{~K}$ ) carried out to investigate the rotational dynamics of the fullerene group in the higher quality solvent-free structure revealed atomic displacement parameters consistent with fast rotation of the highly symmetric fullerene in the solid state, whereas the imidazolium unit remains in a fixed position and therefore represents the stator. DFT and semiempirical calculations were applied to get insight into the profile of the rotational potential of the fullerene unit, particularly considering interactions with the neighboring molecules in the crystal lattice. The results indicate that the crystal environment leads to the presence of one lowest energy minimum that is connected to seven others that are slightly higher in energy through rotational barriers of approximately $1.5-2.5 \mathrm{kcal} \mathrm{mol}^{-1}$.
\end{abstract}

\section{Introduction}

A detailed understanding of molecular dynamics in the solid state is essential to develop the working principles of smart materials and artificial molecular machines. ${ }^{1}$ One of the most promising strategies to document the relation between molecular structure and condensed phase dynamics relies on the design and characterization of crystalline molecular rotors. $^{2-5}$ Molecular architectures that enable rotation in the solid state take advantage

\footnotetext{
${ }^{a}$ Center for Polymers and Organic Solids, Departments of Chemistry \& Biochemistry and Materials, University of California, Santa Barbara, California 93106, USA. E-mail: bazan@chem.ucsb.edu

${ }^{b}$ Department of Chemistry and Biochemistry, University of California, Los Angeles, California 90095, USA. E-mail: mgg@chem.ucla.edu

${ }^{c}$ Departament de Química Física \& Institut de Química Teòrica i Computacional (IQTCUB), Universitat de Barcelona, Spain

$\dagger$ Electronic supplementary information (ESI) available: Thermal analysis of solvent-free $\{\mathbf{1}\}$ and density functional theory and semiempirical calculations of the rotational potential in the gas phase and in the solid state. Crystal structure data for $\{\mathbf{1}\}(100 \mathrm{~K}, 80-480 \mathrm{~K}),\left\{\mathbf{1}(\mathrm{tol})_{2}\right\}$, and two refinements of $\left\{\mathbf{1}(\mathrm{CNPT})_{2}\right\}$. CCDC 986384-986396.
}

¥ Present address: Institut für Anorganische Chemie, Julius-MaximiliansUniversität Würzburg, Am Hubland, 97074 Würzburg, Germany. E-mail: andreas.lorbach@uni-wuerzburg.de of structural elements that create free volume, such as bulky stators that can shield the rotator, ${ }^{2,4,5}$ and an alternative that works well even in relatively dense, close-packed systems, is based on the use of rotators with a high-order symmetry axis and a regular shape. ${ }^{6}$ The higher the symmetry of the rotator, the greater the number of energy minima per period, the smaller the angular displacement between adjacent rotational states, and the smaller the energy barriers that interconnect them. ${ }^{6}$ In that context, it is known that single crystals of $\mathrm{C}_{60}$ display an isotropic rotary cubic phase above $250 \mathrm{~K} .^{7,8} \mathrm{In}$ $\mathrm{C}_{60}$-cubane heteromolecular crystals $\left(\mathrm{C}_{60} \cdot \mathrm{C}_{8} \mathrm{H}_{8}\right)$, cubane acts as a stator, and free rotation of the fullerene rotator is observed as low as $150 \mathrm{~K}$; the fullerene-fullerene distance is greater in the heteromolecular crystal than in the pure $\mathrm{C}_{60}$ rotary cubic phase. ${ }^{9}$ Molecular dynamics simulations indicate that the axis of rotation of the fullerene changes randomly, while the cubane stator vibrates in place. ${ }^{10}$ Tour et al. took advantage of the pronounced rotational flexibility of $\mathrm{C}_{60}$ when designing a nanoscale car which contained four fullerene wheels that were connected to the molecular backbone by single point attachments governed by the formal 1,2-addition of an alkyne $\mathrm{C}-\mathrm{H}$ bond to a double bond of $\mathrm{C}_{60} \cdot{ }^{11-13}$

One of our groups recently developed the fullerene derivative $\mathbf{1}$ (Scheme 1), ${ }^{14}$ which may be viewed as a crystalline molecular rotor 


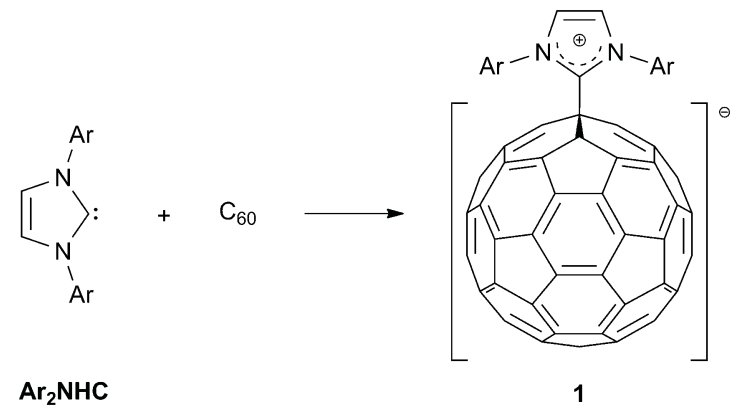

Scheme 1 Synthesis of the fullerene-carbene adduct $1(\operatorname{Ar}=2,6$ diisopropylphenyl)

with a single point of attachment and an open topology. Compound 1 is readily available by reaction of $\mathrm{C}_{60}$ with the corresponding aryl-substituted N-heterocyclic carbene 1,3-bis(2,6-diisopropylphenyl)imidazol-2-ylidene $\left(\mathbf{A r}_{2} \mathbf{N H C}\right)$ by a Lewis acid-base reaction. ${ }^{14}$ Characterization of 1 by single crystal $\mathrm{X}$-ray diffraction in a solvent-containing specimen confirmed the molecular connectivity, but a detailed discussion of the structure and its rotational dynamics was not possible due to insufficient quality of the data. ${ }^{14}$ To some extent this problem could be attributed to the presence of highly disordered solvent molecules, which hamper structural refinement. Such a propensity of fullerenes to form clathrates is demonstrated by the fact that the solid-state structure of solvent-free $[6,6]$-phenyl- $\mathrm{C}_{61}$-butyric acid methyl ester (PCBM) has been reported just recently despite its widespread use, e.g., as an electron acceptor in organic photovoltaic devices. ${ }^{15}$ The determination of the structure relied in this case on powder specimens which were freed from clathrated ortho-dichlorobenzene. ${ }^{15}$

In this contribution we describe a procedure that provides access to high quality solvent-free single crystals of $\mathbf{1}$, which allow for a detailed structural analysis by X-ray diffraction studies as a function of temperature. The variable temperature (VT) data provide the basis to estimate the rotational potential and dynamics of the fullerene rotator by analyzing the anisotropic displacement parameters (ADPs) and by computational modeling. We describe here a surprisingly structured rotational potential with one preferred crystallographic site and up to seven other energy minima per period, despite the nearly spherical shape of the $\mathrm{C}_{60}$ rotator. Notably, small energy barriers of only 1.5-2.5 kcal mol ${ }^{-1}$ were consistently estimated from both VT X-ray analysis and computational methods.

\section{Results and discussion}

\section{Crystallization of solvent-free 1}

Several crystallization conditions had to be systematically explored to form single crystals of $\mathbf{1}$ that did not include solvent molecules. The best result was achieved when a $0.76 \% \mathrm{w} / \mathrm{w}$ solution of $\mathrm{C}_{60}$ in dry 1-chloronaphthalene (CNPT; contained approx. 10\% 2-chloronaphthalene) was treated with an equimolar amount of 1,3-bis(2,6diisopropylphenyl)imidazol-2-ylidene ( $\mathbf{A r}_{2} \mathbf{N H C}$ ) at room temperature under inert conditions ( $c f$. the ESI $\dagger$ for experimental details).
At room temperature a microcrystalline precipitate is formed after several hours indicating an oversaturation of the product mixture. To better control the crystallization of $\mathbf{1}$, the reaction time was limited to $10 \mathrm{~min}$ and the resulting optically clear purple solution was filtered to remove potential seed crystals. The filtrate was then immediately diluted with dry toluene (toluene/CNPT $7: 1$ by volume) and stored at room temperature to give solvent-free crystals $\{\mathbf{1}\}$ (up to $1 \mathrm{~mm}$ in size) along with two concomitant solvates that contain toluene $\left(\left\{\mathbf{1}(\mathrm{tol})_{2}\right\}\right)$ and 1-chloronaphthalene $\left(\left\{\mathbf{1}(\mathrm{CNPT})_{2}\right\}\right)$. The occurrence of different clathrates within the same crystallization vessel is reproducible, and attempts to optimize the crystallization conditions such that exclusively $\{\mathbf{1}\}$ is formed failed. However, through a process of visual inspection and measurement of lattice parameters by means of XRD experiments, it was determined that solvent-free crystals can be recognized within a given batch, as they tend to be more elongated than those of the two clathrates. The structure obtained from a solvent-free crystal $\{\mathbf{1}\}$ at $100 \mathrm{~K}$ was refined in the orthorhombic space group Pnma to an $R=4.09 \%$ (Fig. 1). Diffraction data from $\left\{\mathbf{1}(\mathrm{tol})_{2}\right\}$ obtained at $100 \mathrm{~K}$ were refined in the monoclinic space group $P 2_{1} / c$ with an $R=5.19 \%$ and two crystallographically different toluene molecules in the lattice. Similarly, crystals of $\left\{\mathbf{1}(\mathrm{CNPT})_{2}\right\}$ examined at $130 \mathrm{~K}$ were refined in the space group $P \overline{1}$ with two distinct molecules of CNPT and a relatively large $R=10.6 \%$. Due to the high disorder the metrical parameters of $\left\{\mathbf{1}(\mathrm{CNPT})_{2}\right\}$ are not discussed in detail, but can be found in the $\mathrm{ESI} \dagger$ (Table S1 and Fig. S3).

Using the high quality solvent-free structure of $\{\mathbf{1}\}$ at $100 \mathrm{~K}$ one can gauge distortions on the $\mathrm{C}_{60}$ cage by looking at the bond lengths surrounding the $\mathrm{C}_{i p s o}$ atom, i.e., $\mathrm{C} 15$ in Fig. 1. ${ }^{16-19}$ In crystals of unsubstituted $\mathrm{C}_{60}$ at $110 \mathrm{~K}$, the average length of $\mathrm{C}-\mathrm{C}$ bonds shared by two six-membered rings is 1.399(7) $\AA$ $\left(d_{6,6}\left(\mathrm{C}_{60}\right)\right)$, while the average length of $\mathrm{C}-\mathrm{C}$ bonds shared by a five- and a six-membered ring is 1.445(5) $\AA\left(d_{5,6}\left(\mathrm{C}_{60}\right)\right) .{ }^{19}$ In $\{\mathbf{1}\}$, however, the fullerene $\mathrm{C}_{i p s o}-\mathrm{C}$ bond fusing the two six-membered

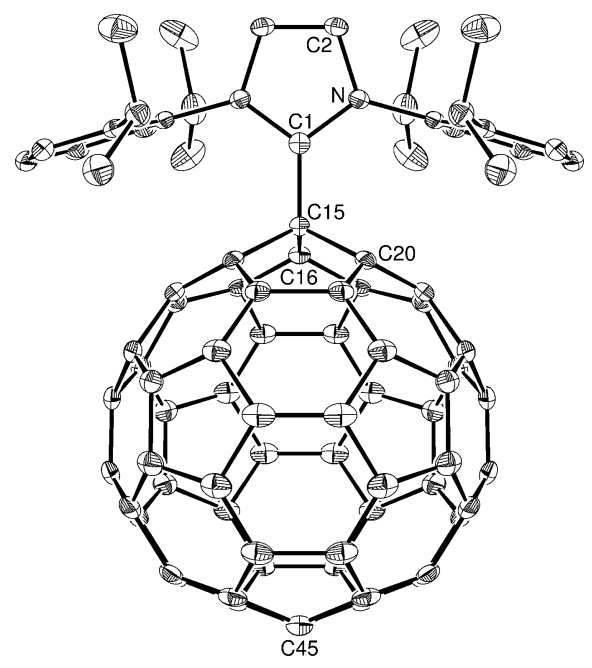

Fig. 1 Molecular structure and numbering scheme in $\{\mathbf{1}\}$; displacement ellipsoids are drawn at the $50 \%$ probability level; hydrogen atoms are omitted for clarity; $T=100 \mathrm{~K}$. 
rings is elongated by $0.133 \AA$ (C15-C16: $1.532(3) \AA$ ). The two equivalent $\mathrm{C}_{i p s o}-\mathrm{C}$ bonds fusing five- and six-membered rings are elongated in $\{\mathbf{1}\}$ by $0.112 \AA$ (C15-C20: 1.557(2) $\AA$ ). These bond lengths, as well as the length of the bond that is formed in the course of adduct formation (C1-C15: 1.530(3) $\AA$ ), indicate the presence of four $\mathrm{C}_{i p s o}-\mathrm{C}$ single bonds. The average lengths of the remaining fullerene $\mathrm{C}-\mathrm{C}$ bonds in $\{\mathbf{1}\}\left(d_{6,6}=1.393(5) \AA\right.$; $\left.d_{5,6}=1.447(6) \AA\right)$ are nearly identical to the same averages in $\mathrm{C}_{60} \cdot{ }^{19} \mathrm{~A}$ comparison of the distances between antipodal carbon atoms (i.e., C15 ‥C45, Fig. 1) indicates a displacement of $\mathrm{C} 15$ away from the fullerene center by $0.35 \AA$ (Table S2, ESI $\dagger$ ). This structural change can be attributed to the changes in the bond lengths and angles due to the tetracoordination of C15 as a result of the attachment of the NHC.

A structural comparison of the imidazolium fragment in $\{\mathbf{1}\}$ with the parent "free" carbene $\mathbf{A r}_{2} \mathbf{N H C}$ reveals only minor changes in the key structural parameters. The most significant effect of the adduct formation is an increase of the $\mathrm{N}-\mathrm{C}-\mathrm{N}$ bond angle from $101.4^{\circ}$ in $\mathbf{A r}_{2} \mathbf{N H C}^{20}$ to $106.5(2)^{\circ}$ in $\{\mathbf{1}\}$ and a decrease of the $\mathrm{N}-\mathrm{C} 1$ bond length by $0.015 \AA$ upon adduct formation, which is qualitatively also true for $\left\{\mathbf{1}(\mathrm{tol})_{2}\right\}$ (Fig. S1, ESI $\dagger$ ). We consider this to be a consequence of the decreased electron density at $\mathrm{C} 1$ resulting in a reduced repulsion with the nitrogen centered electron pairs. This loss of repulsive electronic interactions within the five-membered ring allows a conformation in which the bond angles within the imidazolium fragment in $\{\mathbf{1}\}$ are more equalized than in the free carbene, i.e., they reach values that are close to the angle of an ideal pentagon $\left(108^{\circ}\right)$. The same trend can be observed in the structure of the protonated carbene 1,3-bis(2,6-diisopropylphenyl)- $1 H$-imidazol-3-ium bromide. As in $\{\mathbf{1}\}$, in this imidazolium salt the free electron pair of the $\mathrm{N}$-heterocyclic carbene is involved in a $\sigma$-type bond which results in bond angles within the imidazolium scaffold close to $108^{\circ} .^{21}$ These higher precision structural metrical parameters of 1 , relative to previous reports, are entirely consistent with the originally proposed description of $\mathbf{1}$ as a Lewis acid-base adduct of the acidic $\mathrm{C}_{60}$ and the basic carbene. ${ }^{14}$

In order to describe the rotational orientation of the $\mathrm{C}_{60}$ moiety, one can take the plane of the static imidazolium unit of 1 as the frame of reference. We define the angle $\varphi$ by considering the dihedral angle between this plane (shown in red in Fig. 2A) and the $\mathrm{C}_{i p s o}-\mathrm{C}$ bond fusing the two six-membered rings in $\mathrm{C}_{60}$ (e.g., C15-C16 in $\{\mathbf{1}\}$ ). Using this convention one finds that $\varphi=$ $90^{\circ}$ for $\{\mathbf{1}\}$. However, examination of the solvates $\left\{\mathbf{1}(\mathrm{tol})_{2}\right\}$ and $\left\{\mathbf{1}(\mathrm{CNPT})_{2}\right\}$ reveals that $\varphi=1.1(9)^{\circ}$ and $\varphi=64.2(3)^{\circ}$, respectively. ${ }^{22}$ It is therefore reasonable to suspect that the energy differences between various rotamers should be small, such that structures with $0^{\circ} \leq \varphi \leq 90^{\circ}$ could be present in solution and in the solid state.

\section{Comparison of solvent-free and solvate lattices}

A clear structural difference between $\{\mathbf{1}\}$ and the solvate $\left\{\mathbf{1}(\text { tol })_{2}\right\}$ becomes apparent when their packing patterns are compared (Fig. 2B vs. 2C). Due to the complexity of the structure we only discuss the positions and the relative orientations of the fullerene fragments, defined by the center of gravity (COG) of the
A)

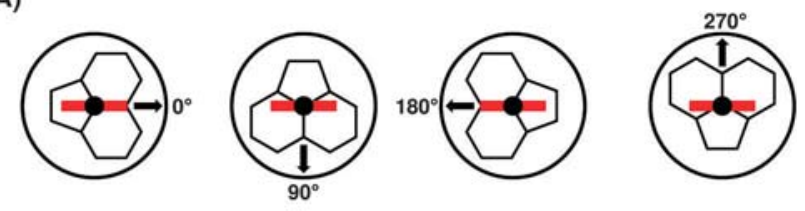

B)
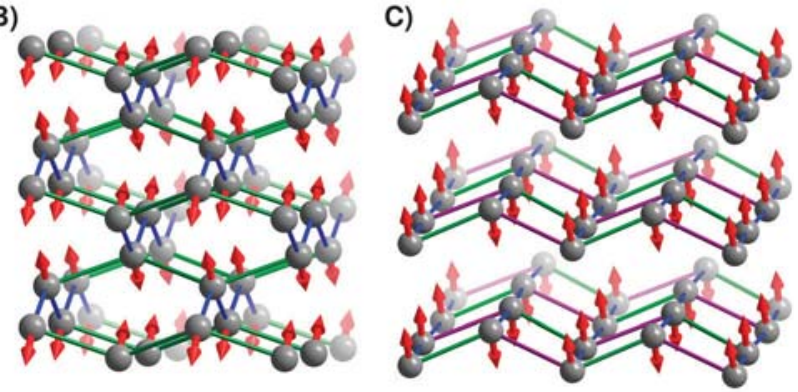

interfullerene distances

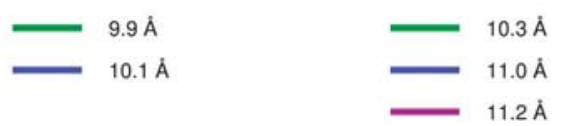

Fig. 2 Schematic representation of the rotamers of 1 with angles $\varphi$ of $0^{\circ}$, $90^{\circ}, 180^{\circ}$, and $270^{\circ}$ (A). Packing diagrams of $\{\mathbf{1}\}$ (B) and $\left\{\mathbf{1}(\mathrm{tol})_{2}\right\}$ (C). Each sphere represents the center of gravity of a fullerene unit; the arrows indicate the orientation of the molecule defined as $\mathrm{C}_{\text {ipso }} \rightarrow \mathrm{C} 1$.

60 carbon atoms of each fullerene unit and the $\mathrm{C}_{i p s o} \rightarrow \mathrm{C} 1$ vector. As shown in Fig. $2 \mathrm{~B}$, solvent-free $\{\mathbf{1}\}$ exhibits a packing structure that can be described as a three dimensional framework. Each of the $\mathrm{C}_{60}$ moieties is surrounded by four neighbors (interfullerene COG-COG distances below $12 \AA$ ), which are arranged in a distorted tetrahedral geometry (angles between the centers of the fullerenes $\alpha=2 \times 86.8^{\circ}, 2 \times 109.5^{\circ}, 130.7^{\circ}$, and $133.4^{\circ}$ ). One pair of equidistant fullerenes is located at a distance of $9.9 \AA\left(\alpha=133.4^{\circ}\right)$, the other pair has a distance of $10.1 \AA\left(\alpha=130.7^{\circ}\right)$. In contrast to the solvent-free crystal, $\left\{\mathbf{1}(\mathrm{tol})_{2}\right\}$ exhibits a two dimensional structure of parallel pleated fullerene layers in which each fullerene is surrounded by four fullerenes in a distorted square planar geometry $\left(\alpha=72.9^{\circ}\right.$, $76.4^{\circ}, 102.2^{\circ}, 108.4^{\circ}, 127.9^{\circ}$, and $\left.174.8^{\circ}\right)$. The interfullerene distances to the four neighbors are longer than in $\{\mathbf{1}\}$ and the two equidistant neighboring fullerene units (11.0 $)$ are almost linearly aligned $\left(\alpha=174.8^{\circ}\right)$, while the angle between the closest fullerene neighbor $(10.3 \AA)$ and the more distant one $(11.2 \AA)$ is equal to $\alpha=127.9^{\circ}$. We anticipate that the interactions between the molecules of compound $\{\mathbf{1}\}$ are mainly achieved by $\pi-\pi$ as well as dipole-dipole interactions considering that in $\mathbf{1}$ the fullerene moiety bears a negative partial charge and that the imidazolium fragment is positively charged. ${ }^{14}$

\section{Variable temperature diffraction experiments}

The fact that the fullerene structure in $\{\mathbf{1}\}$ is not rotationally disordered at low temperature indicates that it sits at a welldefined energy minimum, and that any rotational motion must occur through discrete Brownian jumps, rather than by a 
diffusive (barrierless) mechanism. ${ }^{23}$ To investigate the rotational dynamics of the fullerene in the solid state we set out to examine its rotational potential by computational methods and to document its rotational dynamics by analysis of the single crystal X-ray diffraction-determined anisotropic displacement parameters (ADPs), also known as thermal ellipsoids, as a function of temperature.

Single crystal X-ray diffraction data of solvent-free $\{\mathbf{1}\}$ were acquired between $80 \mathrm{~K}$ and $480 \mathrm{~K}$ in $50 \mathrm{~K}$ steps (Cooler: Cryostream Plus from Oxford Cryosystems). The low temperature structures $(T \leq 280 \mathrm{~K})$ refined to $R$ factors between $4.2 \%$ and $4.9 \%$ (Fig. 3A). The higher temperature structures $(T \geq 330 \mathrm{~K})$ refined to $R$ values between $6.4 \%$ and $8.3 \%$ (Table S1, ESI $\dagger$ ). Visual analysis of the thermal ellipsoids as a function of temperature confirms that thermal activation results in an increasingly greater delocalization of electron density for all the atoms in the structure. The magnitude and preferential elongation direction for the atoms of the $\mathrm{C}_{60}$ portion suggest that atoms near the equator of the sphere undergo large displacements from their equilibrium positions, while the electron density of the atoms near the poles C15 and C45 remain relatively localized, suggesting that angular rotation occurs about the axis formed by the $\mathrm{C} 1-\mathrm{C} 15$ bond.

For a first investigation of the molecular dynamics we examined the equivalent isotropic displacement parameter $U_{\text {eq }}$ (derived from the ADPs: $U_{\text {eq }}=\left(U_{11}+U_{22}+U_{33}\right) / 3$ ) of each atom to test whether the disorder of the fullerene unit at

A)

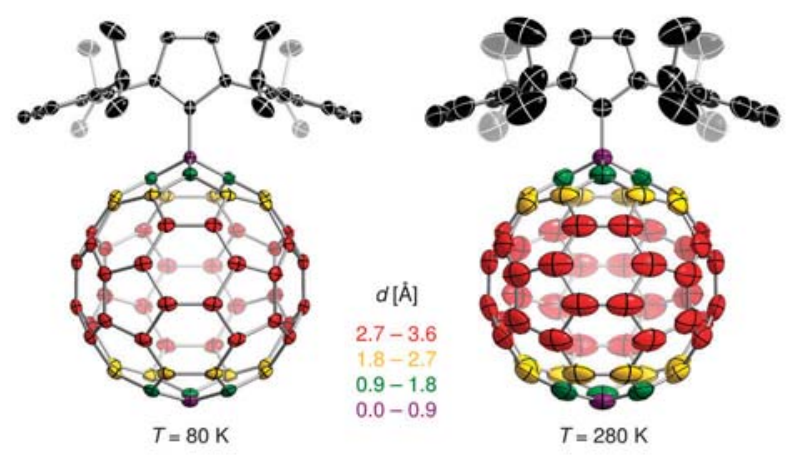

B)

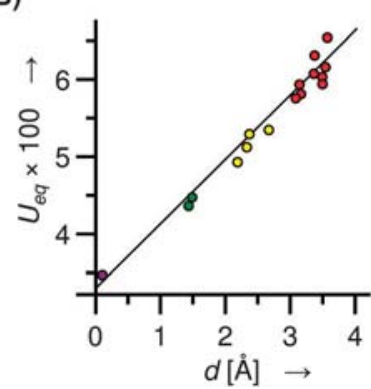

C)

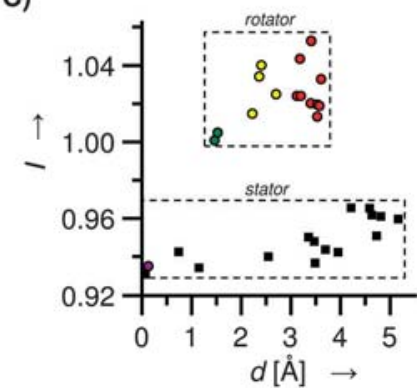

Fig. 3 (A) Displacement ellipsoids (60\% probability level) at $T=80 \mathrm{~K}$ (left, $R=4.4 \%$ ) and $280 \mathrm{~K}$ (right, $R=4.9 \%$ ) and color code. (B) Correlation between the equivalent isotropic displacement parameter $\left(U_{\text {eq }}\right)$ and the distance $d$ of an atom to the fullerene-imidazolium axis at $T=$ 280 K. (C) Relative increase I of $U_{\text {eq }}$ vs. $d$ (cf. Formula S1, ESI $\dagger$ for the definition of $I$; circles: fullerene atoms; squares: imidazolium atoms).
$T=280 \mathrm{~K}$ was also in line with a wheel-like rotational flexibility along the C1-C15 axis. Therefore we analyzed $U_{\text {eq }}$ with respect to the distance $d$ of the atom to the assumed rotational axis (defined by the C1-C15 bond). The $U_{\text {eq }}$ vs. $d$ plot (Fig. 3B) indicates a positive correlation between the two parameters which is in line with the proposed model of a molecular rotor.

To determine whether the observed disorder of the fullerene fragment is predominantly of static or dynamic nature, it is more telling to analyze the relative change of $U_{\text {eq }}$ as a function of $T$. We therefore introduced the normalized relative increase $I$ of $U_{\text {eq }}$ and calculated this parameter for all non-hydrogen atoms of $\{\mathbf{1}\}$ ( $c f$. Formula S1, ESI $\uparrow$ for the definition of $I$; the data obtained at $T=480 \mathrm{~K}$ were not considered due to large error margins). The $I v s$. $d$ plot indicates the presence of two groups of atoms (Fig. 3C; for the sake of clarity data of atoms which are symmetry equivalent or placed on antipodal points on the fullerene sphere were averaged). All atoms of the imidazolium stator, as well as the two fullerene atoms that are located on the assumed rotational axis, belong to the group with $0.93<I<0.97$. The remaining fullerene atoms show larger $I$ values $(1.00<I<1.06)$, which we take it to imply a stronger temperature dependence of their displacement parameters and therefore a more dynamic nature of disorder.

\section{Thermal motion analysis}

To analyze the ADPs in terms of a rotational energy profile we used the Thermal Motion Analysis program (THMA14C) written by Schomaker and Trueblood, ${ }^{24,25}$ as adapted in WinGX by Farrugia. ${ }^{26} \mathrm{~A}$ model that accounts for translation, libration, and screw motion (TLS model) is obtained from the atomic displacement tensors with a least-squares procedure. The fullerene atoms plus $\mathrm{C} 1$ were treated as an isolated molecule in the TLS model (ESI $\dagger$ ). With this model, the principal amplitude of libration $(L 1)$ of $\mathrm{C}_{60}$ is found to be about the axis of least inertia, close to the C1-C15 axis. $L 1$ is $5.3\left({ }^{\circ}\right)^{2}$ at $80 \mathrm{~K}$, while $L 1$ in the same direction for the entire molecule is $3.8\left(^{\circ}\right)^{2}$. (The smaller $L 2$ and $L 3$ amplitudes about the perpendicular inertial axes suggest a torsional potential (ESI $\dagger$ ), in which $L 1$, or $\left\langle\Phi^{2}\right\rangle$, is a measure of the width of the potential well.) The mean-square amplitude of libration, $\left\langle\Phi^{2}\right\rangle$, was determined at $50 \mathrm{~K}$ intervals from $80 \mathrm{~K}$ to $480 \mathrm{~K}$. As illustrated in Fig. $4,\left\langle\Phi^{2}\right\rangle$, for the fragment $\mathrm{C}_{60}+\mathrm{C} 1$, is larger than that for the molecule as a whole at all temperatures and increases more rapidly above $330 \mathrm{~K}$. However, the magnitude of $\left\langle\Phi^{2}\right\rangle \approx 30\left({ }^{\circ}\right)^{2}$ at $300 \mathrm{~K}$ is surprisingly small, suggesting thermal oscillations with an amplitude of $c a$. $5^{\circ}$. Considering the spherical shape of the $\mathrm{C}_{60}$ fragment, one may conclude that the rotator oscillates in a surprisingly narrow energy well!

\section{Rotational potential}

In order to gain insight into the internal dynamics of 1 we investigated the rotational potential by computational modeling methods. Simple symmetry considerations indicate that the rotation of $\mathrm{C}_{60}$ relative to the imidazolium fragment should give rise to energetic variations with several maxima and minima, as different atoms from the fullerene come into contact with the atoms from the reference NHC group. While the gas phase 


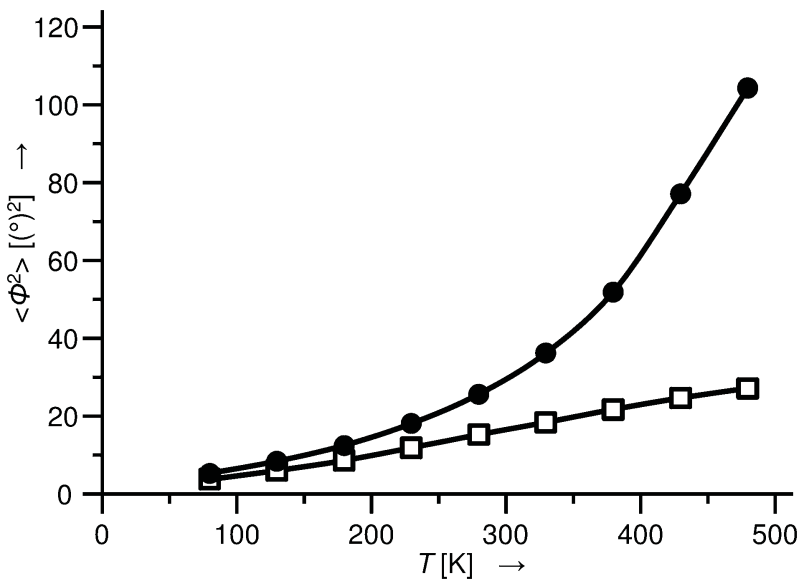

Fig. 4 Mean-square amplitude of libration $\left\langle\Phi^{2}\right\rangle$ as a function of temperature $T$ (THMA14C). The filled circles correspond to the $\mathrm{C}_{60}+\mathrm{C}_{1}$ fragment and the open squares to the entire molecule 1.

structure of the imidazolium moiety should be 2-fold symmetric, the imidazolium heterocycle and its packing environment in the crystal have mirror, but not 2-fold, symmetry. We expected these symmetries to be reflected in the corresponding gas phase and solid state potentials. To determine that, we utilized density functional theory (DFT) based methods with increasing capabilities to account for polarization effects and non-bonding interactions. Based on the good agreement between calculated and experimentally determined structures, the M06-2X/6-31G(d,p) method was selected (Table S8, ESI $\dagger$ ). To determine the gas phase M06-2X potential, the energy was minimized with respect to all molecular degrees of freedom except for the angle that defines the torsional potential, i.e., $\varphi$. Single point energy calculations for 80 different torsion angles between $0^{\circ}$ and $360^{\circ}$ (i.e., $4.5^{\circ}$ increments) were then used to generate the potential curve (Fig. 5A). This method reveals rotational barriers smaller than ca. $1.8 \mathrm{kcal} \mathrm{mol}^{-1}$. The results identify the angle observed in the $\mathrm{X}$-ray structure of $\{\mathbf{1}\}$ as the lowest energy minimum at symmetry equivalent angles of $\varphi=90^{\circ}$ and $\varphi=270^{\circ}$. The potential energy curve reveals the expected 2-fold symmetry with degenerate features related by $180^{\circ}$, which is characterized by two sets of five minima per period.

Crystal packing effects on the rotational energy were analyzed with a cluster model consisting of a single molecule $\mathbf{1}$ embedded in the cavity created by its nearest neighbors in the solvent-free $100 \mathrm{~K}$ crystal structure, which only included atoms within a radius of about $5 \AA$ from the surface. The free valences of these fragments were saturated with $\mathrm{H}$-atoms (Fig. S13, ESI $\dagger$ ). In order to obtain a potential energy curve in the crystal environment comparable to that calculated for the isolated molecule, we performed similar single point energy calculations allowing all degrees of freedom of the molecule to relax except for $\varphi$, while all the atoms of the cluster used to simulate the crystal environment remained in their original positions during the scan.

Given the large size of the model ( 125 atoms in the reference molecule and 593 atoms in its environment) we were not able to treat it by means of computationally demanding DFT methods
A)

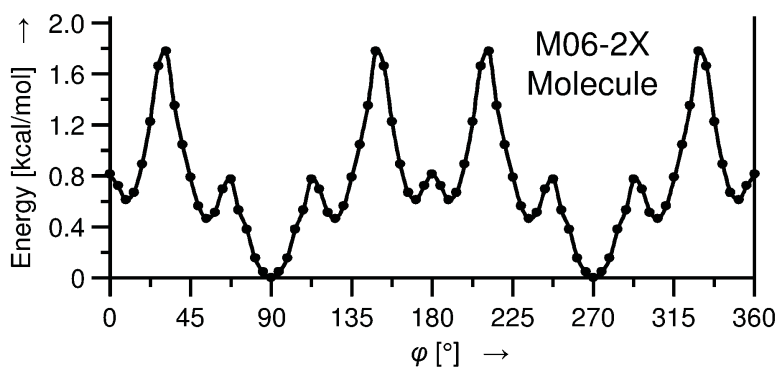

B)

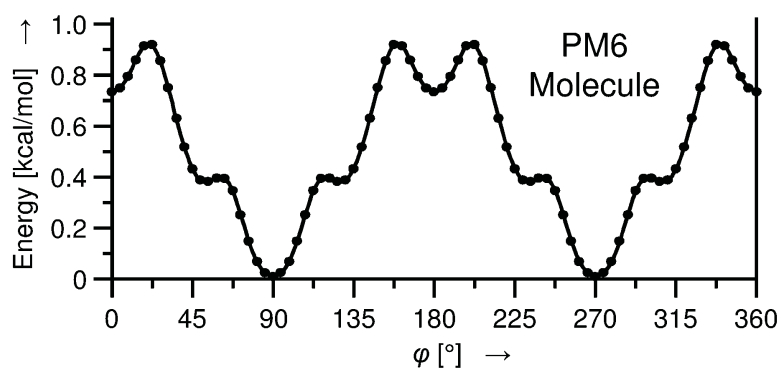

C)

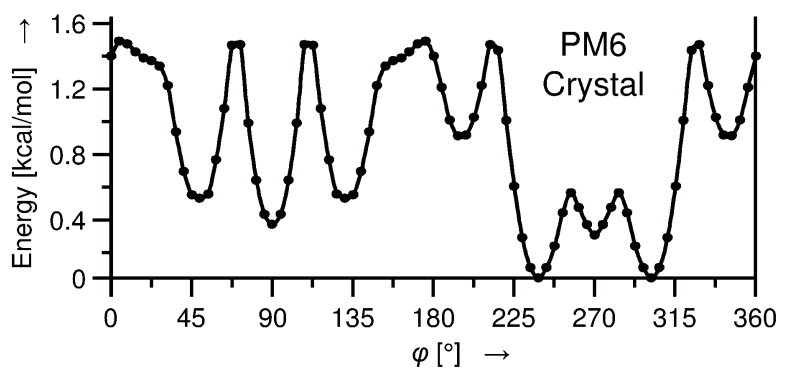

Fig. 5 Fullerene rotational potential in adduct 1 for isolated molecules in the gas phase with the M06-2X functional (A) and the PM6 method (B), and for a cluster derived from the crystal structure of solvent-free $\{\mathbf{1}\}$ at $100 \mathrm{~K}$ with the PM6 method (C).

and it was necessary to limit the theoretical level to semiempirical methods. In general, such methods can not reliably describe weak non-covalent interactions, e.g., van der Waals forces. Thus, we first evaluated various semiempirical methods (AM1, PM3, and PM6) in order to choose one which describes the particular system in the best possible way. The M06-2X energy profile obtained for the isolated molecule 1 (Fig. 5A) was used as the benchmark and we found that the PM6 method showed the best correlation between the two energy profiles (Fig. 5B and Fig. S12, ESI $\dagger$ ) and was therefore used for the estimation of the rotational potential in the solid state. The main differences between the M06-2X and PM6 potentials are the greater heights of all the features in the former, and the lack of a small barrier at $0^{\circ}$ and $180^{\circ}$ for the latter. However, the shape of the profile, which is of particular interest in this study, is reproduced well with the PM6 method. ${ }^{27}$ We want to point out that an adequate description of zwitterionic systems is particularly challenging with such methods. This is illustrated by the observed deviations in the local charge distributions compared to those obtained by DFT methods (B3LYP, B97D, and M06-2X; $c f$. Table S10, ESI $\dagger$ ). On the other hand, the dipole 
moment and overall charge separation are reproduced well by the semiempirical method PM6 ( $c f$. Table S9, ESI $\dagger$ ). We therefore assume that the applied strategy is a suitable way to get a qualitative insight into the energy profile. However, it has to be emphasized that the accurate calculation of barrier heights for conformational changes in complex systems with many competing weak non-covalent interactions such as in the present case is extremely difficult, and the actual values should be taken just as a qualitative estimation of the order of magnitude of the barriers present in the system.

As expected, the potential energy of the rotator in the cluster is no longer 2 -fold rotationally symmetric. Mirror symmetry in Fig. 5C is readily identified by degenerate features at $90^{\circ} \pm \varphi$ and $270^{\circ} \pm \varphi$. Relatively shallow rotational minima calculated for the isolated molecule become steeper in the cluster as a result of an increase in the size of all the barriers. While energy variations between the various minima and maxima are too small to be considered quantitatively accurate, the multiple narrow wells in the calculated solid state potential account for the unexpected VT X-ray observations.

Variations in the magnitude of the mean square torsional amplitude, $\left\langle\Phi^{2}\right\rangle$, as a function of temperature in Fig. 4 provide key information on the dynamic behavior of the fullerene rotators. $^{24,25}$ While the uncertainty in this number is large, we interpret the trend in $\left\langle\Phi^{2}\right\rangle$ values as a function of increasing temperature in terms of angular displacements that escape the deepest minimum. In general, $\left\langle\Phi^{2}\right\rangle$ values as a function of temperature follow a relatively weak $T^{3 / 2}$ dependence, which is based on Debye theory, over a considerable range. ${ }^{28,29}$ This behavior is observed for the entire molecule over all temperatures (open squares in Fig. 4), and with small deviations for the rotating fragment (dark circles) in the region where the structure can be refined to good $R$ factors (i.e., $R \leq 4.9 \%$ at $80 \mathrm{~K} \leq T \leq 280 \mathrm{~K}$ ). ${ }^{30}$ The large upward deviation above these temperatures approximates an exponential dependence, suggesting that the rotator escapes from its preferred crystallographic site to explore adjacent higher-energy minima for short periods of time, as expected for a temperature-dependent equilibrium process. Indeed, partial occupation of neighboring sites leads to growing electron density detected as larger thermal ellipsoids that change their directionality because the observed electron density comes from carbon atoms in adjacent sites that are located above and below the original latitude ( $c f$. Fig. S5, ESI $\dagger$ ). We note that the mean estimated standard deviation of the isotropic displacement parameter values $[\operatorname{mesd}(U)]$ rises even more steeply than the $\left\langle\Phi^{2}\right\rangle$ values themselves (Fig. S8, ESI $\dagger$ ), supporting the notion of an increasing contribution of positional disorder. Barrier heights $(B)$ are generally estimated by assuming a simple periodic $n$-fold potential, $V=(B / 2)(1-\cos n \Phi)$, where $V$ is the energy as a function of angular displacement about $\Phi$. The larger the rotational symmetry order, $n$, the smaller the angular displacement between neighboring sites. If one considers an 8-fold potential, as suggested by PM6, transition states for rotation occur within $c a .22 .5^{\circ}$ from each minimum and local barriers are expected in the range of 1.5-2.5 $\mathrm{kcal} \mathrm{mol}^{-1},{ }^{31}$ in qualitative agreement with the calculations in Fig. 5C. We note that these rotational barriers are lower than those for the $t \mathrm{Bu}$ group in a comparable fullerene derivative $\mathrm{Li}\left[t \mathrm{BuC}_{60}\right]$ determined by means of variable temperature ${ }^{1} \mathrm{H}$ NMR spectroscopy in THF solution at $-60{ }^{\circ} \mathrm{C}$ to be $\Delta G^{\ddagger}=9.3 \pm$ $0.1 \mathrm{kcal} \mathrm{mol}{ }^{-1} \cdot{ }^{32}$ A different study carried out at 110, 153, and $200 \mathrm{~K}$ found a rotational barrier of more than $8 \mathrm{kcal} \mathrm{mol}^{-1}$ for disordered, unsubstituted $\mathrm{C}_{60}$ in the solid state. ${ }^{19}$ This finding could be attributed to the fact that unsubstituted $\mathrm{C}_{60}$ packs more closely in the solid state leading to shorter intermolecular distances and stronger interactions.

\section{Conclusions}

By virtue of the single bond attachment between the imidazolium fragment and the $\mathrm{C}_{60}$ cage, and the ensuing rotational degree of freedom, one can examine crystals of $\mathbf{1}$ within the context of molecular rotors. A key experimental advance of practical consequence concerns the availability of high-quality, solventfree single crystalline phases, which are difficult to obtain due to the propensity of fullerenes to incorporate solvent molecules in the crystal lattice. Compound $\mathbf{1}$ is a rare example of fullerene derivatives which could be obtained in this study as a highly crystalline solvent-free system $\{\mathbf{1}\}$. This system formed the basis of variable temperature X-ray crystallographic investigations, which provided us with an experimental framework to estimate the energy landscape of rotational dynamics in the solid state. An analysis of the experimentally obtained anisotropic atomic displacement parameters by means of the Thermal Motion Analysis program (THMA14C) in combination with a semiempirical approach (PM6 method) used to calculate the rotational energy of the $\mathrm{C}_{60}$ unit within the solvent-free crystal lattice $\{\mathbf{1}\}$ reveals an energy profile with barriers of 1.5-2.5 $\mathrm{kcal} \mathrm{mol}^{-1}$ and eight sharp energy minima. While barriers of this magnitude are not surprising for a spherically-shaped rotator, its behavior in an energy potential with multiple narrow wells is documented here for the first time. We conclude by pointing out that the rotational motion of semiconducting fragments such as $\mathrm{C}_{60}$ in the solid state is poorly understood and is likely to bear influence on the issue of phonon transport. ${ }^{33,34}$

\section{Acknowledgements}

A.L. wishes to thank the Alexander von Humboldt Foundation for a Feodor Lynen Research Fellowship for postdoctoral researchers. Work at UCLA was supported by NSF grant DMR1101934. Work in Barcelona was supported by the Ministerio de Economía y Competividad (project CTQ2011-23862-C02-02) and the Generalitat de Catalunya (project 2009SGR-1459). The stage of A.C. at UCLA has been financed by a grant from the Ministerio de Economía y Competividad.

\section{Notes and references}

1 M. A. Garcia-Garibay, Nat. Mater., 2008, 7, 431-432.

2 M. A. Garcia-Garibay, Proc. Natl. Acad. Sci. U. S. A., 2005, 102, 10771-10776. 
3 G. S. Kottas, L. I. Clarke, D. Horinek and J. Michl, Chem. Rev., 2005, 105, 1281-1376.

4 T.-A. V. Khuong, J. E. Nuñez, C. E. Godinez and M. A. GarciaGaribay, Acc. Chem. Res., 2006, 39, 413-422.

5 C. S. Vogelsberg and M. A. Garcia-Garibay, Chem. Soc. Rev., 2012, 41, 1892-1910.

6 S. D. Karlen, H. Reyes, R. E. Taylor, S. I. Khan, M. F. Hawthorne and M. A. Garcia-Garibay, Proc. Natl. Acad. Sci. U. S. A., 2010, 107, 14973-14977.

7 P. A. Heiney, J. E. Fischer, A. R. McGhie, W. J. Romanow, A. M. Denenstein, J. P. McCauley, Jr., A. B. Smith, III and D. E. Cox, Phys. Rev. Lett., 1991, 66, 2911-2914.

8 W. I. F. David, R. M. Ibberson, T. J. S. Dennis, J. P. Hare and K. Prassides, Europhys. Lett., 1992, 18, 219-225.

9 S. Pekker, É. Kováts, G. Oszlányi, G. Bényei, G. Klupp, G. Bortel, I. Jalsovszky, E. Jakab, F. Borondics, K. Kamarás, M. Bokor, G. Kriza, K. Tompa and G. Faigel, Nat. Mater., 2005, 4, 764-767.

10 V. R. Coluci, F. Sato, S. F. Braga, M. S. Skaf and D. S. Galvão, J. Chem. Phys., 2008, 129, 064506.

11 Y. Shirai, Y. Zhao, L. Cheng and J. M. Tour, Org. Lett., 2004, 6, 2129-2132.

12 Y. Shirai, A. J. Osgood, Y. Zhao, K. F. Kelly and J. M. Tour, Nano Lett., 2005, 5, 2330-2334.

13 G. Vives and J. M. Tour, Acc. Chem. Res., 2009, 42, 473-487.

14 H. Li, C. Risko, J. H. Seo, C. Campbell, G. Wu, J.-L. Brédas and G. C. Bazan, J. Am. Chem. Soc., 2011, 133, 12410-12413.

15 M. Casalegno, S. Zanardi, F. Frigerio, R. Po, C. Carbonera, G. Marra, T. Nicolini, G. Raos and S. V. Meille, Chem. Commun., 2013, 49, 4525-4527.

16 W. I. F. David, R. M. Ibberson, J. C. Matthewman, K. Prassides, T. J. S. Dennis, J. P. Hare, H. W. Kroto, R. Taylor and D. R. M. Walton, Nature, 1991, 353, 147-149.

17 K. Hedberg, L. Hedberg, D. S. Bethune, C. A. Brown, H. C. Dorn, R. D. Johnson and M. de Vries, Science, 1991, 254, 410-412.

18 S. Liu, Y.-j. Lu, M. M. Kappes and J. A. Ibers, Science, 1991, 254, 408-410.

19 H.-B. Bürgi, E. Blanc, D. Schwarzenbach, S. Liu, Y.-j. Lu, M. M. Kappes and J. A. Ibers, Angew. Chem., Int. Ed. Engl., 1992, 31, 640-643.
20 A. J. Arduengo, III, R. Krafczyk, R. Schmutzler, H. A. Craig, J. R. Goerlich, W. J. Marshall and M. Unverzagt, Tetrahedron, 1999, 55, 14523-14534.

21 M. Berger, N. Auner, T. Sinke and M. Bolte, Acta Crystallogr., Sect. E: Struct. Rep. Online, 2012, E68, 01845.

22 The structures of $\left\{\mathbf{1}(\mathrm{tol})_{2}\right\}$ and $\left\{\mathbf{1}(\mathrm{CNPT})_{2}\right\}$ show disorder and contain two conformers of $\mathbf{1}(91.4 \% / 8.6 \%$ and $78.0 \% / 22.0 \%$, respectively). Only the major conformers are discussed.

23 D. E. Woessner, J. Chem. Phys., 1962, 36, 1-4.

24 J. D. Dunitz, V. Schomaker and K. N. Trueblood, J. Phys. Chem., 1988, 92, 856-867.

25 V. Schomaker and K. N. Trueblood, Acta Crystallogr., Sect. B: Struct. Sci., 1998, B54, 507-514.

26 L. J. Farrugia, J. Appl. Crystallogr., 1999, 32, 837-838.

27 A novel parametrization (PM7) has recently been published after the calculations described in our study were already finished: J. J. P. Stewart, J. Mol. Model., 2013, 19, 1-32.

28 C. C. Wilson, Crystallogr. Rev., 2009, 15, 3-56.

29 T. J. Bastow, S. L. Mair and S. W. Wilkins, J. Appl. Phys., 1977, 48, 494-497.

30 We have been reminded by a reviewer that it is possible, with data collections at several temperatures, to correct the refined $U_{\mathrm{ij}}$ values for the effects of high-frequency internal vibrations, and for temperature-independent disorder: H.-B. Bürgi and S. C. Capelli, Helv. Chim. Acta, 2003, 86, 1625-1640. The corrected ADPs may then be analyzed in terms of temperature-dependent rigid-body motion. The authors suggest several crystal structure determinations over as large a temperature range as possible (preferably between about $10 \mathrm{~K}$ and the melting point of the material). Our equipment does not allow measurements below $80 \mathrm{~K}$, therefore we performed our diffraction studies in the widest temperature range accessible. An analysis with the available data is in progress.

31 E. Maverick and J. D. Dunitz, Mol. Phys., 1987, 62, 451-459.

32 P. J. Fagan, P. J. Krusic, D. H. Evans, S. A. Lerke and E. Johnston, J. Am. Chem. Soc., 1992, 114, 9697-9699.

33 J. H. Seol, I. Jo, A. L. Moore, L. Lindsay, Z. H. Aitken, M. T. Pettes, X. Li, Z. Yao, R. Huang, D. Broido, N. Mingo, R. S. Ruoff and L. Shi, Science, 2010, 328, 213-216.

34 T. M. C. Faro, M. S. Skaf and V. R. Coluci, J. Chem. Phys., 2011, 135, 244510. 